\title{
Novel 500-GHz Band Waveguide Stepped Septum-Type Circular Polarizer with a New High-Accuracy and Very Small Waveguide Flange
}

\author{
Yutaka Hasegawa $^{1}$ (D) $\cdot$ Hiroyuki Maezawa $^{1} \cdot$ Hideo Ogawa $^{1}$ \\ Received: 26 August 2020 / Accepted: 8 October 2020 /Published online: 29 October 2020 \\ (C) The Author(s) 2020
}

\begin{abstract}
A new waveguide stepped septum-type circular polarizer (SST-CP) was developed to operate in the $500-\mathrm{GHz}$ band for radio astronomical and planetary atmospheric observations. In a previous study, we developed a practical SST-CP for the $230-\mathrm{GHz}$ band. However, several issues prevent this device being easily scaled down to the $500-\mathrm{GHz}$ band, such as manufacturing dimensional errors and waveguide flange position errors. In this study, we developed a new waveguide flange with a highaccuracy position determination mechanism and a very small size of $10 \times 10 \mathrm{~mm}$. We also developed a new fabrication technique to obtain very good flatness for the device's blank materials by high-accuracy polishing using a resin fixture. Using these new methods, the manufactured $500-\mathrm{GHz}$ band SST-CP achieved a crosspolarization talk level of better than $-30 \mathrm{~dB}$ at $465-505 \mathrm{GHz}$, a device surface flatness of within $3 \mu \mathrm{m}$, and also the horizontal positioning error of $\pm 3 \mu \mathrm{m}$. These results indicate that the developed $500-\mathrm{GHz}$ band SST-CP has high performance in the high-frequency band, and thus the new manufacturing methods are effective in the $500-\mathrm{GHz}$ band.
\end{abstract}

Keywords Waveguide $\cdot$ Circular polarizer $\cdot$ VLBI $\cdot$ Flanges

Yutaka Hasegawa

s_y.hasegawa@p.s.osakafu-u.ac.jp

Hiroyuki Maezawa

maezawa@p.s.osakafu-u.ac.jp

Hideo Ogawa

ogawa@p.s.osakafu-u.ac.jp

1 Department of Physical Science, Graduate School of Science, Osaka Prefecture University, 1-1,

Gakuen-cho, Nakaku, Sakai, Osaka 599-8531, Japan 


\section{Introduction}

Radio wave observations in the submillimeter wave band are important in radio astronomy and atmospheric observations, which deal with the distribution of minor molecules. Above the $100-\mathrm{GHz}$ band, there are various important molecular emission lines, such as $\mathrm{CO}, \mathrm{CS}, \mathrm{NH}_{3}$, $\mathrm{O}_{2}$, and $\mathrm{O}_{3}$. The very long baseline interferometry (VLBI) observation method is one of the greatest advantages of the radio observations. Using VLBI, we can achieve extremely high angular resolution at the microsecond order. For example, the international VLBI observation project the Event-Horizon Telescope (EHT) obtained the world's first direct imaging of black hole shadows by attaining an angular resolution of 30- $\mu$ s degrees in the $220-\mathrm{GHz}$ band [1,2].

A technique for separating orthogonal polarized waves $(\mathrm{V} / \mathrm{H})$ or left-/right-handed circular polarized (LHCP/RHCP) waves is often required in radio observations. For example, it is necessary to unify the observation polarization planes in observation telescopes to construct VLBI. To realize this, in the receiver system of VLBI observatories, circular polarization separation is performed before digital sampling [3, 4]. However, in submillimeter wave observations over $100 \mathrm{GHz}$, it is technically difficult to directly sample the observed signals $[5,6]$. Therefore, it is essential to use a heterodyne receiver which down-converts the observed signal to a lower frequency, where it is easier to handle. In fact, most submillimeter wave spectroscopic receiver systems used in radio astronomy and planetary atmosphere observations are $4 \mathrm{~K}$ cooled heterodyne receivers using a superconducting SIS-mixer [7-10]. However, as heterodyne detectors employing waveguide circuits are generally sensitive to only one linearly polarized wave, a circular polarization separation and a circular to linear conversion mechanism must be equipped in front of the detectors.

The waveguide stepped septum-type circular polarizer (SST-CP) is the simplest and highest-performance device available for circularly polarized wave separation. The SST-CP is a compact device that works independently, and so it is easy to install into a receiver with relatively low insertion loss. It also exhibits no fluctuation in its high polarization separation ratio because there are no moving parts. Thanks to these excellent features, SST-CP has been widely used in the $\mathrm{cm} / \mathrm{mm}$ band $[3,11,12]$. However, there are very few practical examples of SST-CP above the 100-GHz band [13,14]. Therefore, an orthogonal polarization separator is conventionally used instead of an SST-CP, or a combination of two orthogonal polarized waves using an optical element such as a wire grid [15]. One reason why SST-CPs have not been adopted in the submillimeter band may be because the well-known conventional SST-CP models are extremely vulnerable to manufacturing errors and lack of flatness. The waveguide size in the submillimeter band, such as $1.092 \mathrm{~mm}$ for a standard WR-4.3, is small for milling manufacturing, which makes it easy for fatal characteristic deterioration to occur. Thus, in our past development, we proposed a new SST-CP model that can endure a large manufacturing error and obtain a stable performance in the $230-\mathrm{GHz}$ band [14]. This 230-GHz band SST-CP is installed in the 1.85-m telescope of Osaka Prefecture University [16-18], the NANTEN2 4$\mathrm{m}$ telescope of Nagoya University [19, 20], and the 12-m GreenLand Telescope of AcademiaSinica Institute of Astronomy and Astrophysics in Taiwan, which is an observatory conducting the EHT project $[21,22]$, and is currently used for both single-dish astronomical and VLBI observations.

In this study, we developed a new 500-GHz band SST-CP, which is an improved model of the previous $230-\mathrm{GHz}$ band system. Many important molecular emission lines are located in the 500-GHz band, such as $\mathrm{CO}(J=4-3)$ at $461 \mathrm{GHz}, \mathrm{C}[\mathrm{I}]\left({ }^{3} \mathrm{P}_{1}-{ }^{3} \mathrm{P}_{0}\right)$ at $492 \mathrm{GHz}$ [23-25], $\mathrm{H}_{2} \mathrm{O}$ (ortho), $\mathrm{O}_{2}, \mathrm{O}_{3}$, and $\mathrm{HDO}[26,27]$. In circularly polarized observations of these important 
emission lines, a very high circular polarization separation ratio (cross-polarization talk level, $\mathrm{X}$-pol) of over $20 \mathrm{~dB}$ is often required. However, the waveguide size for measurements in the $500-\mathrm{GHz}$ band is so small, as small as $0.559 \times 0.279 \mathrm{~mm}$, while the manufacturing dimensional error is relatively large at approximately $\pm 10 \mu \mathrm{m}$. Thus, even with the previous 230 GHz SST-CP model, which can endure some dimensional error, it cannot achieve such a high polarization separation ratio, unless several technical improvements are made in the electrical design and manufacturing technology. The areas to be improved are the physical factors limited by the UG-387U/M waveguide flange, which has been the world standard in the submillimeter wave band. These factors are the device minimum size, the position error of the two connected waveguides, the horizontal/vertical axis angle error due to insufficient accuracy of the position determination mechanism [28], and the surface contact failure and higher-mode resonation caused by the low surface accuracy on the dividing surfaces for milling manufacturing [14]. To improve these issues, we realized the following two technological developments:

1. We developed a new waveguide connection flange with a very compact size of $10 \times$ $10 \mathrm{~mm}$ and a low horizontal positioning error of approximately $\pm 3 \mu \mathrm{m}$. With this new flange called the high-accuracy and very small (HAS) flange, the minimum size of a 3port waveguide device is reduced to $10 \mathrm{~mm}^{3}$, which is $1 / 8$ smaller than the size of the UG387 flange, and can eliminate the increase in insertion loss due to circuit length. Also, deterioration of characteristics such as the input return loss and X-pol due to connection position/axis angle error is suppressed.

2. We developed a new high-precision milling manufacturing technology, which includes high-precision surface polishing, to improve the contact surface accuracy of the device dividing section and the flange contact surface. Thanks to this, we can obtain a very uniform surface roughness of $\pm 3 \mu \mathrm{m}$ or better. Furthermore, it is expected that the milling error in the depth direction will be reduced.

We describe the details of the electrical design of the 500-GHz SST-CP in Section 2.1, while in Section 2.2, we describe the details of the new HAS waveguide flange. The high-precision manufacturing details are described in Section 3, and Section 4 shows the measurement results of the frequency characteristics and 3D dimensions of the manufactured 500-GHz SST-CPs.

\section{Design}

\subsection{Design of the 500-GHz SST-CP}

A simulation design of the electrical properties of the 500-GHz SST-CP was performed with Ansys HFSS. The dimensions of the SST-CP were optimized by the two analytical models proposed in [14]. One is a model of an SST-CP alone, hereafter referred to as the "single model." The single model consists of a square-shaped input waveguide connecting to a feed antenna in practice, a stepped septum part that gives a phase difference of $90^{\circ}$ onto two orthogonal polarizations, and two rectangular waveguide output ports connecting to LNAs or detectors. The single model simulation can be used to evaluate input/output return losses, isolation between output ports, and phase delay of the vertical axis linear polarized input wave. In this study, the design target values of the input/output return losses and port isolation are under $-20 \mathrm{~dB}$, which is considered to be sufficiently practical for $440-510-\mathrm{GHz}$ band 
observations. The second simulation model is composed of the single model and the mirror duplicated model which connected directly at the square input port, hereafter referred to as the "mirror model." Using this mirror model, we can evaluate the insertion loss and X-pol talk of the left and right circularly polarized wave transmissions. The design target values of the mirror model are an insertion loss of better than $-1.0 \mathrm{~dB}$ in the $440-510-\mathrm{GHz}$ band, and an Xpol talk of $-30 \mathrm{~dB}$ or better for $460-500 \mathrm{GHz}$, where reduction of reverse polarization noise is strongly required, and $-20 \mathrm{~dB}$ for $440-460$ and $500-510 \mathrm{GHz}$.

Figure 1 shows the optimized dimensional design values of the septum part. In order to suppress the characteristic deterioration (mainly X-pol talk) caused by the gap spacing due to contact failure at the device dividing surfaces on top of the septum shown in [14], we adopted a method of dividing the circuit not at the top of the septum but at the fourth stage of the septum, and the gap spacing was shifted to that part. Each dimension has been optimized with considering to suppress the characteristics deterioration from this gap spacing, except to the septum thickness that was fixed at $0.1 \mathrm{~mm}$ to alleviate manufacturing difficulties. In addition, the two output rectangular waveguides divided in two by the septum were extended as a Y shape in a $45^{\circ}$ direction as viewed from the input waveguide propagation direction. This reduced the increase in insertion loss caused by the unnecessary extended waveguide path length due to the limited minimum device size because of the waveguide flange. The estimated loss reduction using the UG-387 U/M flange, which has a minimum device size of $20 \times 20 \times$ $20 \mathrm{~mm}$ for a 3-port device, is $0.14 \mathrm{~dB}(-0.82$ to $-0.68 \mathrm{~dB})$ at $300 \mathrm{~K}$ and $0.04 \mathrm{~dB}(-0.255$ to $0.215 \mathrm{~dB}$ ) at $4 \mathrm{~K}$, when the material is A6063-T5 with an electrical conductivity of $2.3 \times 10^{7} \mathrm{~S} /$ $\mathrm{m}$ and no surface roughness. In another case using the 10-mm HAS flange described in the next Section 2.2, the estimated loss reduction amount is $0.05 \mathrm{~dB}(-0.43$ to $-0.38 \mathrm{~dB})$ at $300 \mathrm{~K}$ and $0.01 \mathrm{~dB}(-0.127$ to $-0.117 \mathrm{~dB})$ at $4 \mathrm{~K}$.

Figure 2 shows the results from the SST-CP HFSS simulation design, which achieved the abovementioned target values with peaks near $490 \mathrm{GHz}$ for reflection characteristics, port isolation, and X-pol talk. Figure 3 shows two examples of X-pol talk characteristics for different shifted gap spacings on the septum. In this case, the gap thickness Gt is changed by $2 \mu \mathrm{m}$ in the range $2-10 \mu \mathrm{m}$ with gap lengths of GL $=0.10 \mathrm{~mm}$ and $0.20 \mathrm{~mm}$ for the gap spacing simulated model shown in Fig. 3a. In Fig. 3b, a trend of monotonic deterioration with increasing Gt can be seen. In Fig. 3c, the bandwidth of X-pol - $25 \mathrm{~dB}$ is broadened, and even when $\mathrm{Gt}=10 \mu \mathrm{m}$, it remains better than $-20 \mathrm{~dB}$ near $500 \mathrm{GHz}$. These results show that in the shifted gap spacing simulation model, the behavior changes significantly for small changes in GL and Gt. The characteristic deterioration for a traditional manufacturing dimensional error

(a)

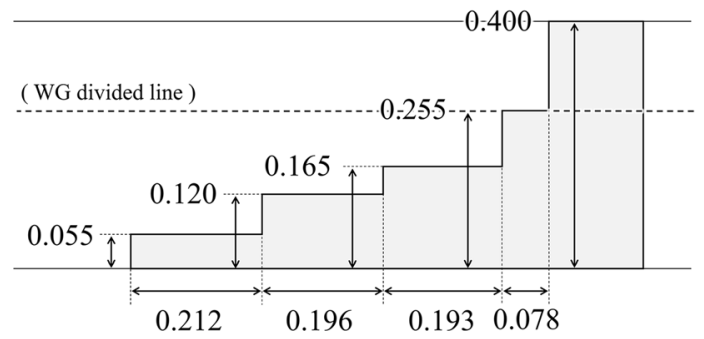

(b)

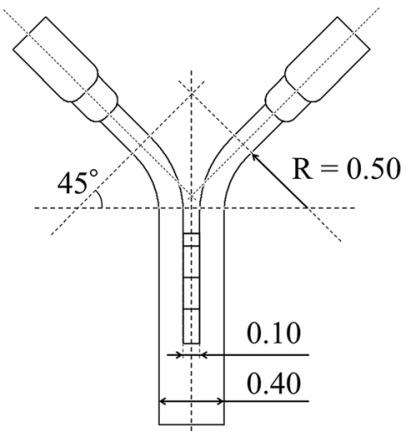

Fig. 1 a Top and $\mathbf{b}$ side view 2D drawing of the 500-GHz SST-CP septum part 
of $\pm 10 \mu \mathrm{m}$ is expected to be suppressed effectively. The simulated X-pol for GL $=0.20 \mathrm{~mm}$ and $\mathrm{Gt}=6 \mu \mathrm{m}$ is better than without a gap, but this is too hard to achieve for manufacturing in the submillimeter band. However, in the centimeter wave band where the manufacturing error is relatively small, it may be possible to achieve better characteristics than previously obtained.

\subsection{High-Accuracy and Small Size Waveguide Flange Design}

The standard waveguide size in the $500-\mathrm{GHz}$ band is as small as W $0.559 \times \mathrm{H} 0.280 \mathrm{~mm}$ (WR2.2 ) or $0.381 \times 0.190 \mathrm{~mm}$ (WR-1.5). As shown in Fig. 1, the total length of the septum part of the $500-\mathrm{GHz}$ band SST-CP is about $0.8 \mathrm{~mm}$. Despite this, a UG-387 waveguide flange with a diameter of $19.5 \mathrm{~mm}$ has generally been used in the submillimeter band, over $100 \mathrm{GHz}$. Therefore, conventional submillimeter band waveguide devices have very long waveguides, resulting in an unnecessary increase of insertion loss. In addition, the alignment mechanism of UG-387 uses four $\varnothing 1.56$-mm knock pins and holes, which are significantly larger than the pins. According to [28], the horizontal positional error of UG-387 is $20 \mu \mathrm{m}$, and the waveguide vertical/horizontal axis angle error is $<1.4^{\circ}$. For SST-CP, an axis angle error of $1.4^{\circ}$ of the input square-shaped waveguide corresponds to $-34-\mathrm{dB}$ X-pol talk level, which cannot satisfy the design target value of $-30 \mathrm{~dB}$.

For these reasons, the UG-387 waveguide flange is clearly insufficient for the $500-\mathrm{GHz}$ SST-CP. Therefore, we developed a new, very small waveguide flange with a highly accurate position determination mechanism, called the high-accuracy and very small (HAS) flange. Figure 4 shows a connection surface 2D drawing and 3D CAD images of the HAS flange. The flange consists of two flange pairs, male and female, like coaxial SMA connectors. The vertical and horizontal positions are determined by highly accurate manufactured position
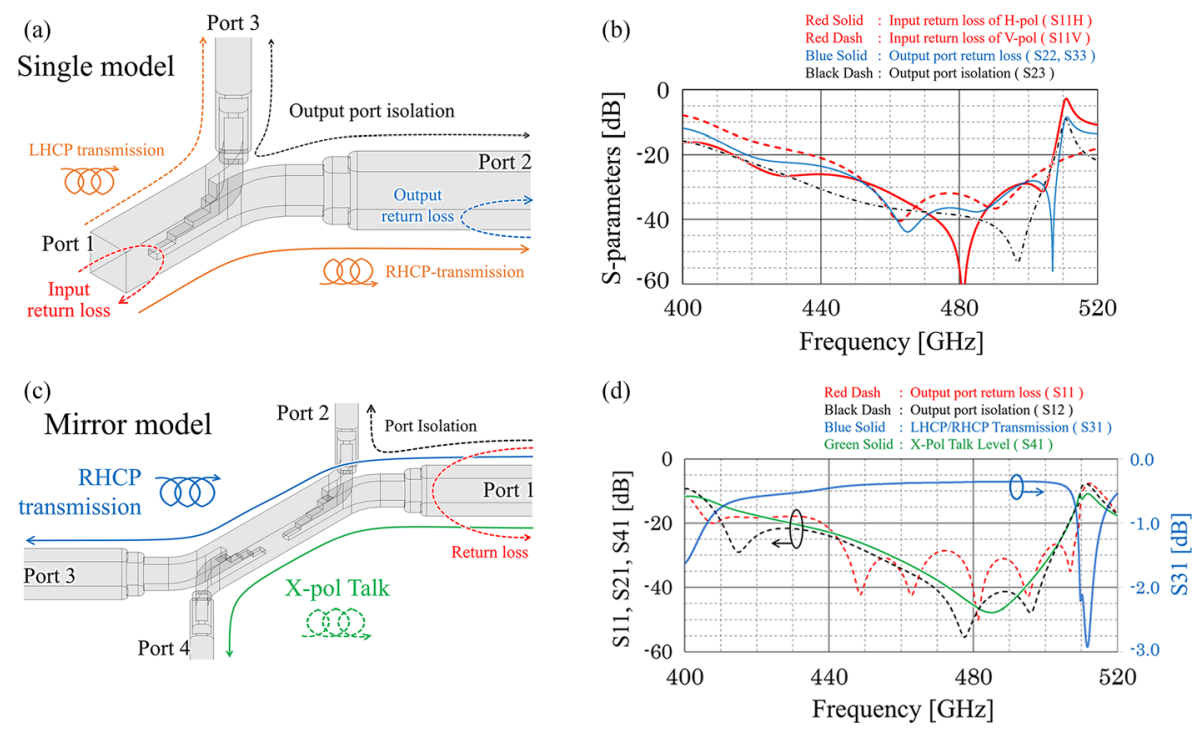

Fig. 2 Results of the 500-GHz SST-CP simulation design. a Schematic of the 500-GHz SST-CP single model and $\mathbf{b}$ its simulated performances; the red solid and dashed lines are V/H-pol input wave return losses, the black line is the output port isolation, and the blue line shows the output port return loss. c Schematic of the mirror duplicated model and $\mathbf{d}$ its simulated performances; the red dashed line is the output port return loss on the mirror model, the blue line is the insertion loss on two connected SST-CPs, the black line is the output port isolation on the mirror model, and the green line shows the X-pol talk level caused by the two duplicated septa 
(a)

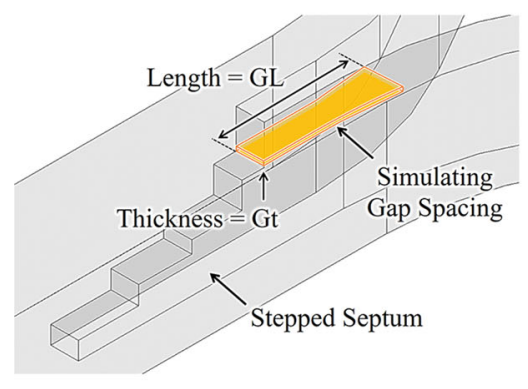

(b) $\mathrm{GL}=0.1 \mathrm{~mm}$

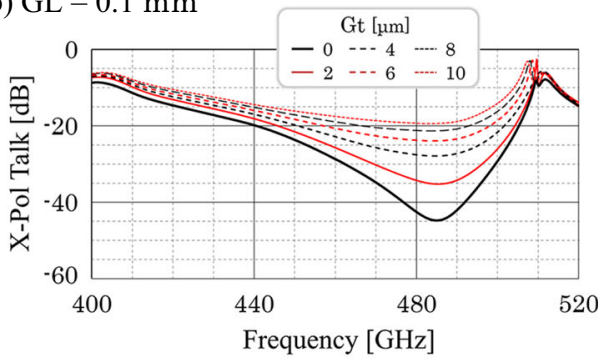

(c) $\mathrm{GL}=0.2 \mathrm{~mm}$

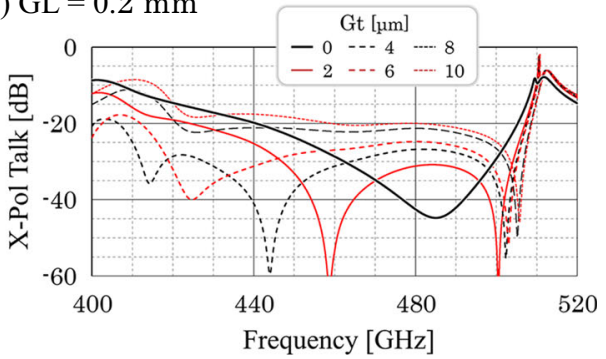

Fig. 3 Simulated X-pol talk deterioration for the SST-CP mirror model with various gap spacings at the 4th step on the stepped septum. a Schematic of the simulation model of the SST-CP with a gap spacing. b X-pol talk performance for various gap thicknesses Gt for a fixed gap length of GL $=0.1 \mathrm{~mm}$ and $\mathbf{c}$ a fixed $\mathrm{GL}=0.2 \mathrm{~mm}$

reference side surfaces on each flange, shown by the $\mathrm{V}$ and $\mathrm{H}$ labels on the yellow protrusions/ depressions in Fig. 4. Thus, the positioning accuracy and axis angle error are determined by the horizontal dimensional milling accuracy and the perpendicularity of the manufactured surfaces. The horizontal dimensional error in our machining is $\sim 3 \mu \mathrm{m}$, so that the HAS flange is several times more accurate than the UG-387. Furthermore, the influence of the offset error and scale error of the milling machine is suppressed by ensuring the milling dimensional origin of the HAS flange pair coincides with each other.

\subsection{0-GHz Band SST-CP Structure Design}

A new positioning mechanism for the HAS flange is also applied in the two-divided SST-CP structures connection position determination, as shown in Fig. 5. Using this method, we expect to be able to suppress the deterioration of the X-pol level due to displacement of the squareshaped input waveguide of the SST-CP core.

Figure 5 shows a top view 2D drawing of the two divided 500-GHz band SST-CP structures. To determine their assembly positions, the HAS flange positioning technique is applied. We thereby expect to be able to suppress the deterioration of the X-pol level caused by the displacement of the square-shaped input waveguide of the SST-CP core. In addition, using the HAS flange allows the SST-CP device size to be reduced to 12 -mm width $\times 10$-mm depth $\times 10$-mm height, which is about $1 / 6$ smaller than the $20 \mathrm{~mm}^{3}$ size when the UG-387 is used. Because of this reduction in device size, the ohmic loss of the waveguide, which is large in the higher frequency band, is greatly reduced. It is also expected that the gap spacing on the septum-divided surfaces is suppressed due to small area ratio between the septum and body. The SST-CP width of $12 \mathrm{~mm}$ is larger than the HAS of $10 \mathrm{~mm}$ so as to avoid 
(a)

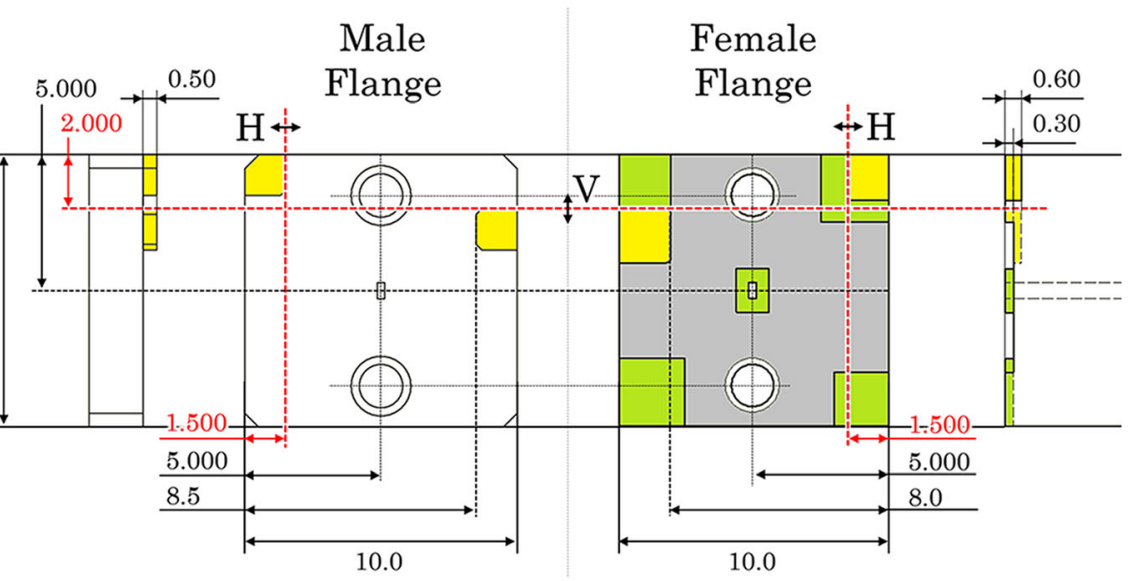

(b)

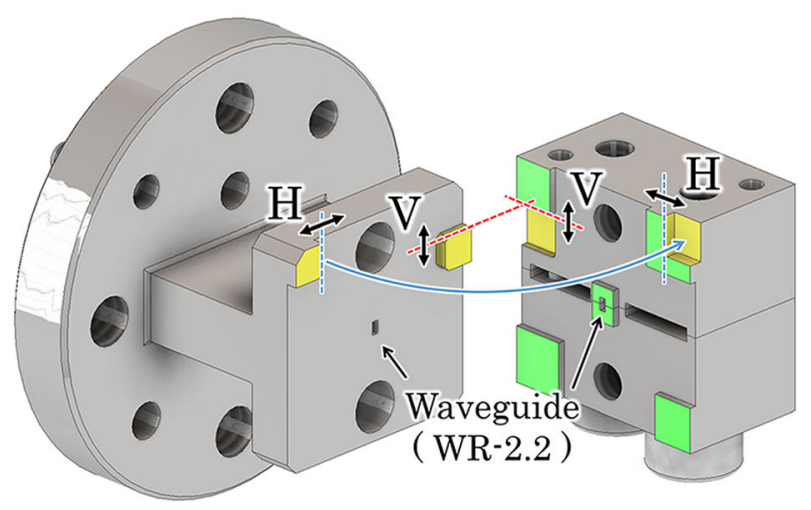

Fig. 4 Schematics of the newly developed HAS flange. a 2D drawing of the connection surfaces on the male and female flange. b 3D CAD image of the HAS flange connection

interference between the left and right pairs of M2 screws that fasten the HAS flange. If a screw smaller than M2 is used, the width can be reduced to $10 \mathrm{~mm}$; however, we did not need to minimize the size in this stage of the development, so we choose M2 screws because of their sufficiently strong fastening force.

In addition, when a special waveguide flange is used, such as HAS, it is necessary to consider how to realize frequency characteristic measurements using the high-frequency vector network analyzer (VNA). In this study, we planned to make measurements using the WR-2.2 waveguide VNA extender module of Virginia Diode Inc. (VDI), which has a standard waveguide port with a UG-387 flange, so a flange converter between HAS and UG-387 was required. Furthermore, two waveguide terminators were required to perform measurements. There were no problems with these, because they could be manufactured separately, as shown in Fig. 6. On the other hand, since it is difficult to measure the frequency characteristics of SST-CP alone, we had to also measure the SST-CP mirror model characteristics. It was expected that it would be difficult to screw-fasten the squareshaped waveguide surface of two SST-CPs to each other using M2 screws, and thus we used bolt-nut fastening for that purpose. 


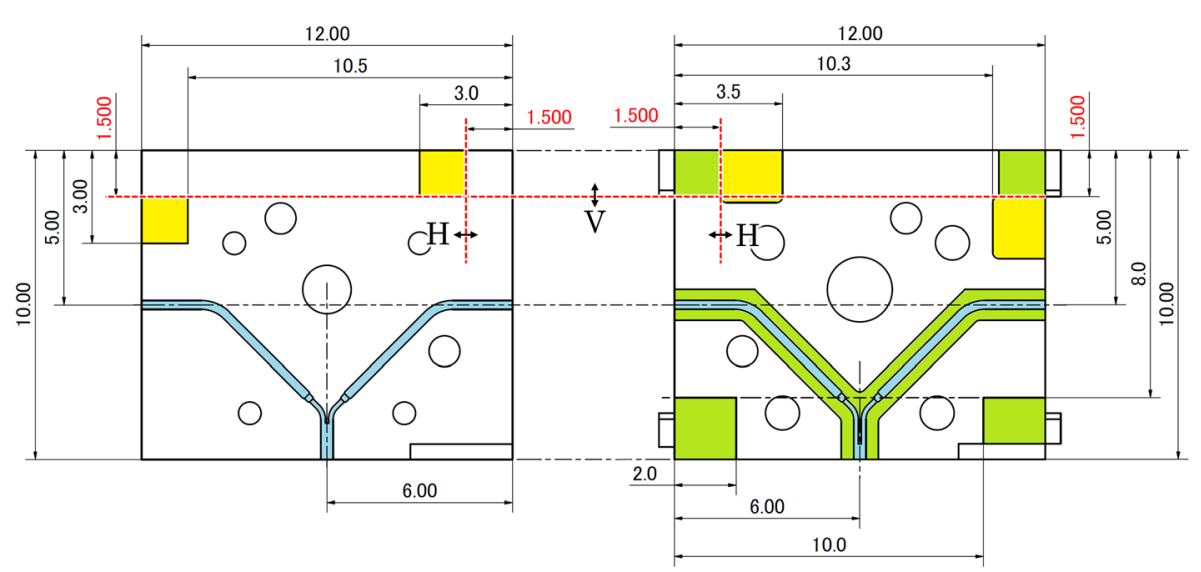

Fig. 5 Top view 2D drawing of the two divided 500-GHz SST-CP structures. Yellow parts, position determination structures; green parts, two-divided parts connection surfaces; blue parts, SST-CP waveguide circuit

\section{Manufacturing with High-Precision Polishing}

Figure 7 shows a photograph of the manufactured 500-GHz SST-CP and companions, as illustrated in Fig. 6. These were manufactured with A6063-T5 aluminum alloy by highprecision machining manufacturing at Kawashima Manufacturing Co., Ltd. (KMCO), with a practical milling accuracy of $\sim 3 \mu \mathrm{m}$ in the horizontal direction and $\sim 5 \mu \mathrm{m}$ in the vertical direction. The A6063-T5 is a superior material and is well matched to the high-precision machining in this study, because of the following five factors: (1) It is known that the surface accuracy of the milling machining is superior than many other metal alloys. (2) The surface accuracy of electrical discharge machining for scraping the waveguide of the flange converter

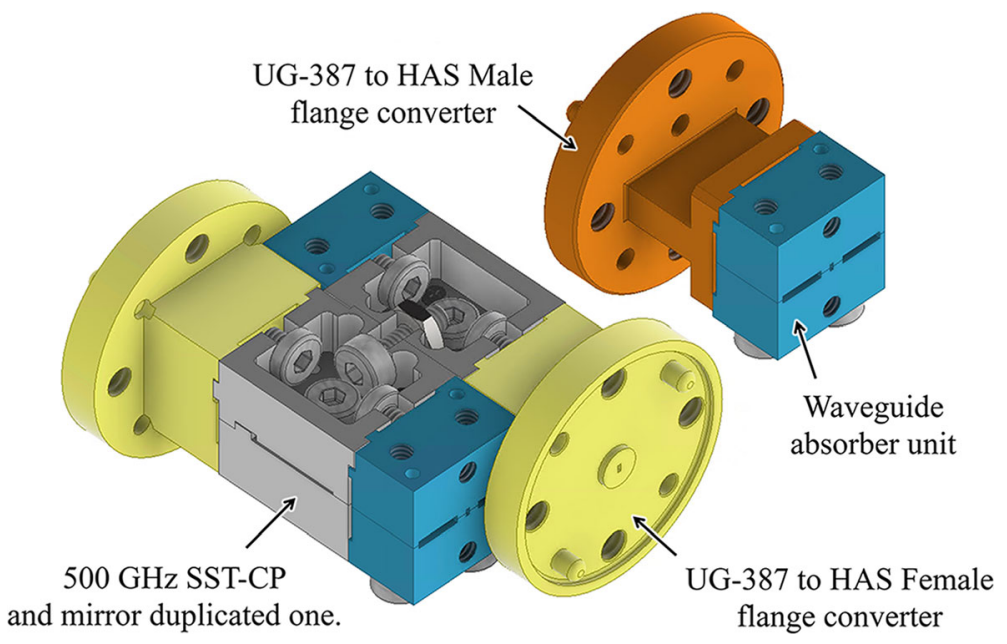

Fig. 6 Schematic of the 500-GHz SST-CP measurement assembly 3D CAD design. Yellow, UG-387 to HAS female flange converter for SST-CP; gray, SST-CPs (a unit and a duplicate mirror unit); blue, waveguide absorber; orange, UG-387 to HAS male flange converter for absorber measurement 


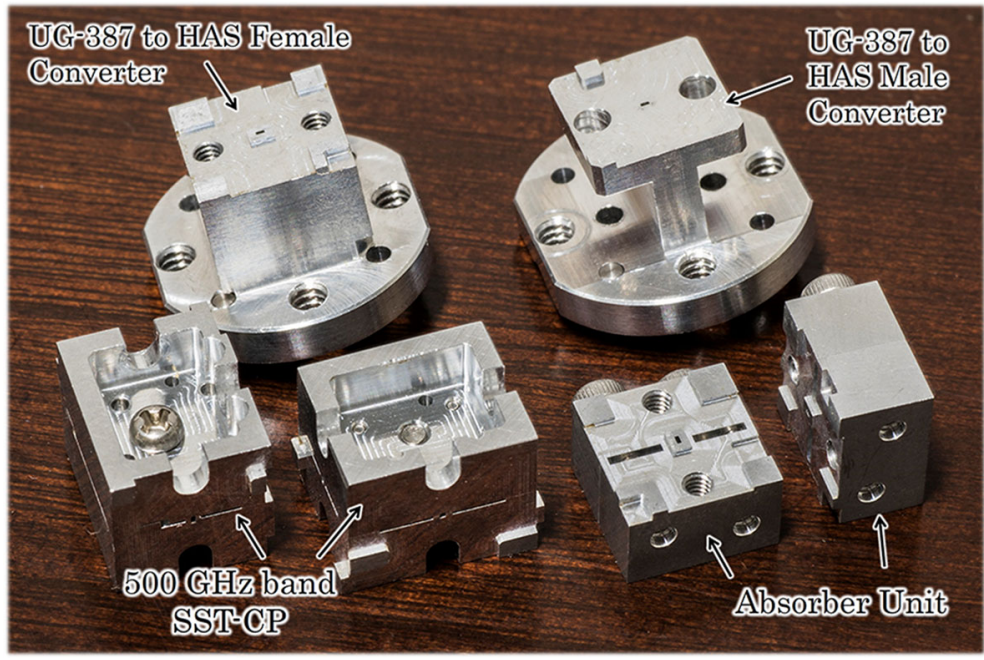

Fig. 7 Photograph of the manufactured 500-GHz SST-CP, waveguide absorber, and an HAS female to UG-387 flange converter

is very good. (3) Since aluminum alloy generally has high weathering and corrosion resistance, it does not require surface plating, such as gold plating on tellurium copper. (4) Among aluminum alloys, A6063-T5 has a uniquely high electrical conductivity, giving a low ohmic loss (0.55 IACS for typical A5052: 0.3, A6061: 0.42). (5) Applying T5 treatment, which is a heat treatment by air cooling after rolling, it can be procured with a relatively low residual stress strain. Therefore, it is not necessary to release stress by annealing before manufacturing.

Furthermore, for high-precision milling fabrication, it is necessary that the orthogonality and surface flatness of the blank material to be fabricated are high. This is because, when the material has poor surface flatness or an angle error in the machining horizontal surface, the machining dimensional error and waveguide connection position error due to the flange contact face angle shift deteriorate. In addition, when the milled surface flatness is poor, the error from the dimensional reference in the depth direction is directly linked to the fabrication error. Therefore, to improve the manufacturing dimensional accuracy, it is important to ensure the orthogonality and flatness of the blank material as well as improving the machining accuracy.

In this study, to obtain high-quality blank materials, we performed high-accuracy surface polishing using a hard resin fixture, after making a squared cutout by general six-face milling. In this method, the cutout blank materials are inserted into just-fitting holes provided in the resin fixture, as shown in Fig. 8. They are then pressed mechanically, together with the resin fixture, onto a lapping table to polish the blank materials at the same contact angle for each surface. The resin fixture was manufactured with very highprecision machining by $\mathrm{KMCO}$, such that we had to insert the blank materials into the fixture holes by hitting them with a wooden hammer, which ensured they could not be easily moved. Using this technique, the six-plane orthogonality of the blanks could be made almost perfect, the flatness of each face could be made extremely high, and we could control the polishing dimension precisely. In this study, the polishing was performed using a liquid compound containing a minimum of $1 \mu \mathrm{m}$ of abrasive particles, and the flatness of the polished surface was $\sim 3 \mu \mathrm{m}$ r.m.s in practice. 


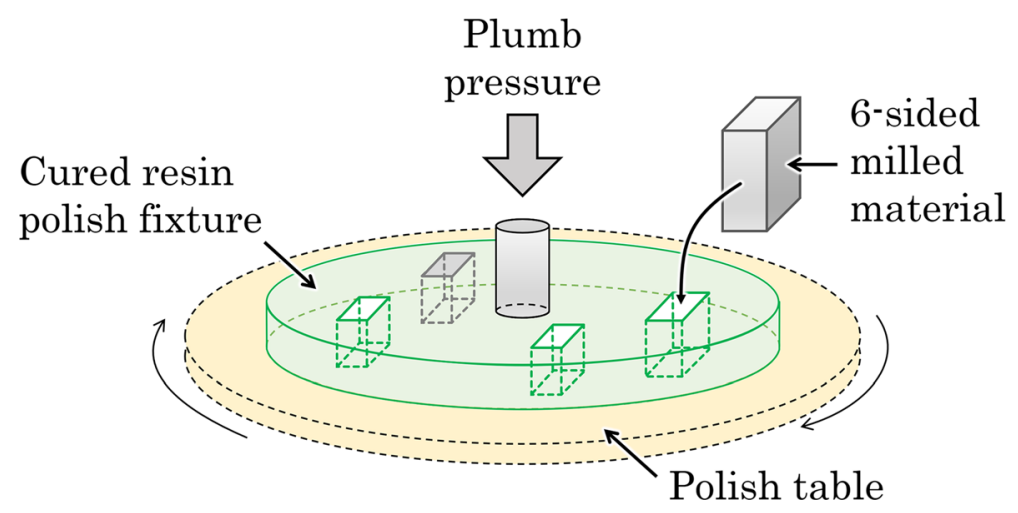

Fig. 8 Schematic of the high-accuracy polishing method with the cured resin fixture

\section{Measurements}

\subsection{Measurement Results for the 500-GHz Band SST-CP}

Figure 9 shows the measured insertion loss of the 500-GHz WG-CPs, and Fig. 10 shows the measured and simulated X-pol talk levels. Figure 11 shows a photograph of the measurement experiment. In this measurement, we used a high-frequency VNA N5260 from Keysight Technologies Inc. and VDI's WR-2.2 waveguide VNA extender module described in Section 2.3, which are owned by the National Institute of Information and Communications Technology (NICT).

The measured LHCP/RHCP insertion losses shown as red and black solid lines in Fig. 9 are very close to the simulation design performances shown by the green dotted line. However, a ripple of about $1-\mathrm{GHz}$ frequency and $\pm 0.1-\mathrm{dB}$ magnitude are evident. This is a standing wave with a wavelength of approximately $15 \mathrm{~cm}$, and considering that the SST-CPs are approximately $10 \mathrm{~mm}$ in size, it is considered that this wave is due to the measurement system and not the device performance.

The measured X-pol talk level shown as the red solid line in Fig. 10 is better than $-30 \mathrm{~dB}$ at 465-505 GHz and $-20 \mathrm{~dB}$ at 435-505 GHz. This result shows that this SST-CP achieves

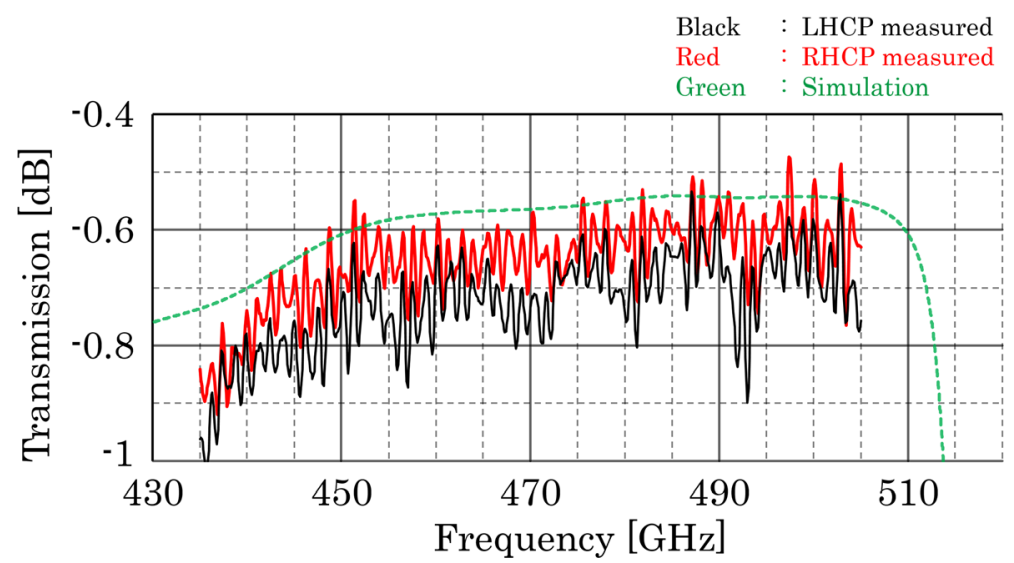

Fig. 9 Measured and simulated insertion losses of the 500-GHz SST-CPs 


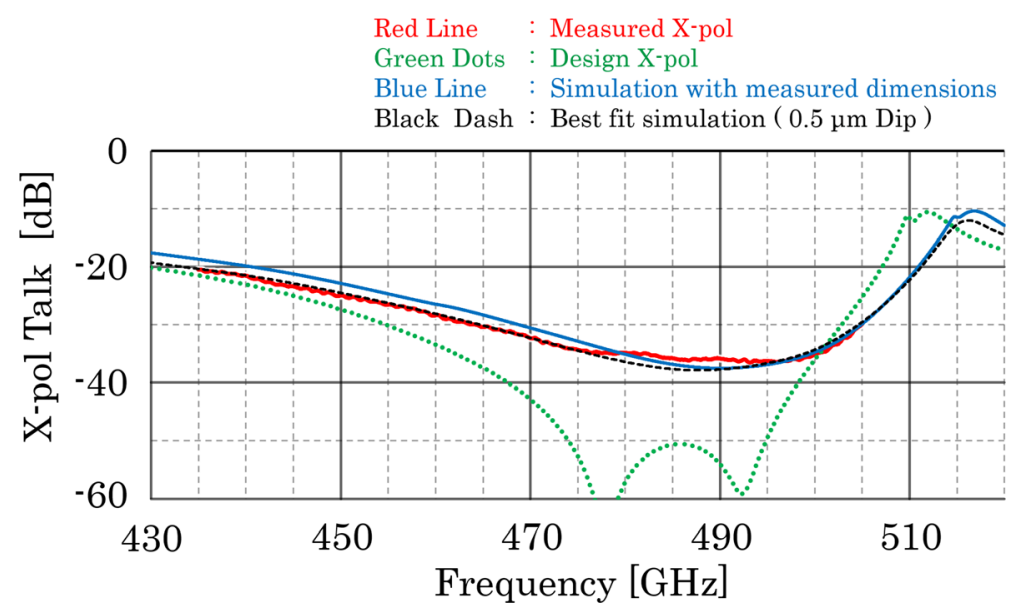

Fig. 10 Measured, simulated, and re-calculated X-pol talk level of the 500-GHz SST-CPs

extremely good cross-polarization separation characteristics in the high-frequency band of $500 \mathrm{GHz}$. However, a difference from the simulated design performance can be recognized, shown as the green dotted line. This is considered to be largely due to manufacturing dimensional error, because the difference between the measured and re-calculated performance using the measured dimensions of manufactured SST-CP shown as the blue solid line is very small. Also, the higher-order mode resonance of $\mathrm{TE}_{11}$ at $510 \mathrm{GHz}$ is shifted to the highfrequency side by about $5 \mathrm{GHz}$.

Furthermore, we estimated the size of the gap spacing on the 4th step of the septum by simulating this gap using the SST-CP analysis model with the measured dimensions. We found a gap length of GL $=0.2 \mathrm{~mm}$ and thickness $\mathrm{Gt}=0.5 \mu \mathrm{m}$ were the best fit, shown as the black dashed line. This result suggests that polishing with the resin fixture significantly improved the flatness of this device-divided surface, giving a flatness of $0.5 \mu \mathrm{m}$ compared to the conventional value of over $10 \mu \mathrm{m}$, such for the $230-\mathrm{GHz}$ SST-CP.

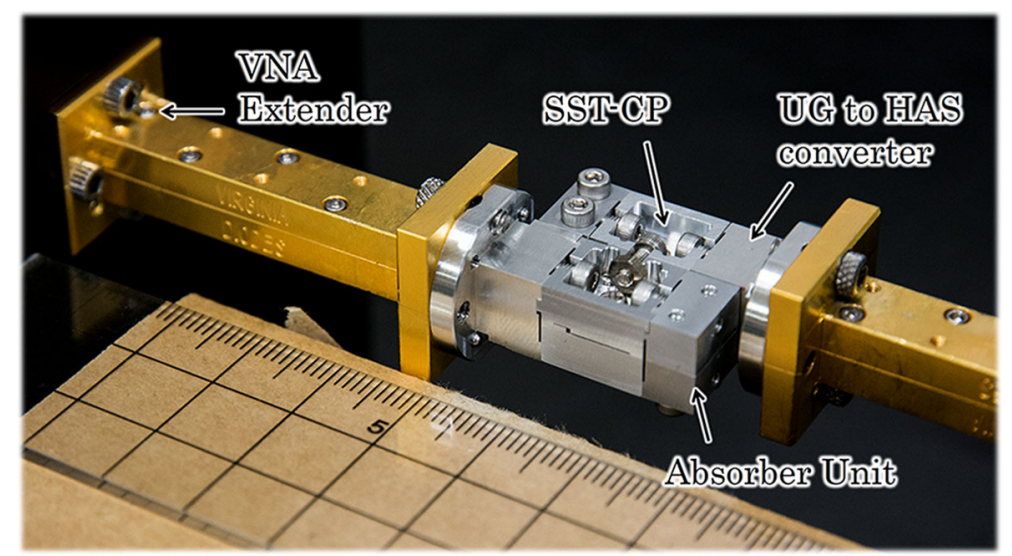

Fig. 11 Photograph of the performance measurement experiment of the 500-GHz band SST-CP with the VDI WR2.2 VNA extender module 


\subsection{High-Accuracy Small Size WG-Flange VNA-Measured Result}

Figures 12 and 13 show the fluctuation of the measured input return losses due to waveguide flange connection errors. In this measurement, two pairs of identical straight waveguides with UG-387 and HAS flanges were clamped, measured, and disassembled seven times for UG-387 and 10 times for the HAS flange. The results for the UG-387 flange in Fig. 12 show that the input return loss magnitude varies over a fairly wide range of -14 to $-26 \mathrm{~dB}$, and the frequency characteristics also change significantly. In the result for the HAS flange, shown in Fig. 13, the trend of the frequency characteristics is almost unchanged, and it can be seen that the magnitude error is very small, at approximately $1 \mathrm{~dB}$. From these results, it is considered that the HAS flange position determination mechanism by the positioning side surface obtained by high-precision machining is very effective, at least in the $500-\mathrm{GHz}$ band. Moreover, it can be expected to be effective even in the higher frequency bands, such as the $1-\mathrm{THz}$ band where the waveguide size is halved.

\subsection{Surface Accuracy Measured Result}

Figures 14 and 15 show the results of high-accuracy 3D measurements of the side surface of the waveguide absorber (4.6) and the SST-CP two-divided surface (4.7). These are examples of polished surfaces using a resin fixture. In the 3D measurements, we used Mitaka Koki NH$6 \mathrm{~N}$, which has a measurement accuracy of $0.1 \mu \mathrm{m}$, at the Instrument Development Center, Graduate School of Science, Nagoya University. From Fig. 14, it can be said that the surface flatness obtained by polishing with a resin fixture is distributed in the range of 0 to $+1.5 \mu \mathrm{m}$, and the surface roughness is $0.6 \mu \mathrm{m}$ r.m.s, which is very uniform. It should be noted that this measurement surface includes the effect of a permanent strain due to vice gripping during machining. Figure 15 shows that the surface accuracy of the divided surface of the SST-CP is $\pm 3 \mu \mathrm{m}$. In particular, it is extremely flat, at almost $0 \mu \mathrm{m}$, near very important points such as the septum contact part and square-shaped input port. This result is in good agreement with the estimated gap spacing thickness Gt of $0.5 \mu \mathrm{m}$ shown in Fig. 10.

However, in Fig. 15, a dent of up to $-3 \mu \mathrm{m}$ can be seen near the right and left rectangular output waveguides, and a bulge of up to $+2.1 \mu \mathrm{m}$ can be seen near the upper M2 fastening screw hole. The following two factors can be considered as causes of such unevenness: (1)

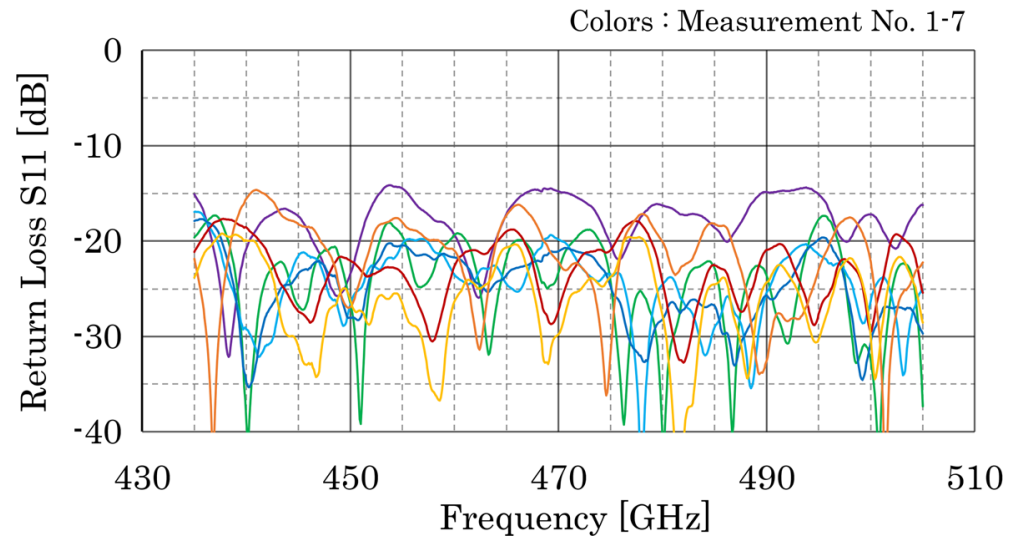

Fig. 12 Measured input return losses of a straight waveguide using a UG-387 flange, reassembled seven times 


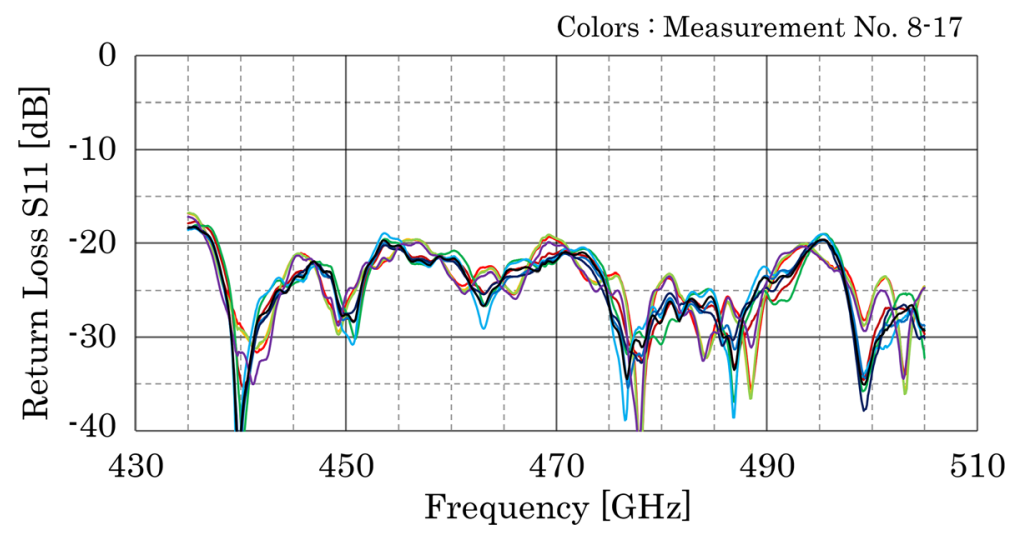

Fig. 13 Measured input return losses of a straight waveguide using an HAS flange, reassembled 10 times

stress strain of the blank material fixing vise during machining and (2) distortion due to pulling of the M2 fastening screw. The stress strain is considered at the time of manufacture, and the manufacturing is performed so that such strains are suppressed near the SST-CP core. However, it is not possible to eliminate the effect of these strains on the entire material and thus when further suppression is required, it is necessary to develop another method such as holding vacuum attraction. The effect of the M2 screw is a known issue, and the influence on the contact accuracy can be largely avoided by digging (anti-cocking method) near the screw hole, though it cannot be reduced to zero. It may be possible to mitigate this effect by controlling the screw tightening torque with a torque wrench or a similar tool.

\section{Conclusion}

In this paper, we developed a high-performance waveguide septum-type circular polarization separator WG-SST-CP which can achieve a cross-polarization talk level (X-pol) of better than $-30 \mathrm{~dB}$ in the $500-\mathrm{GHz}$ band where observation demands are very high in radio astronomy

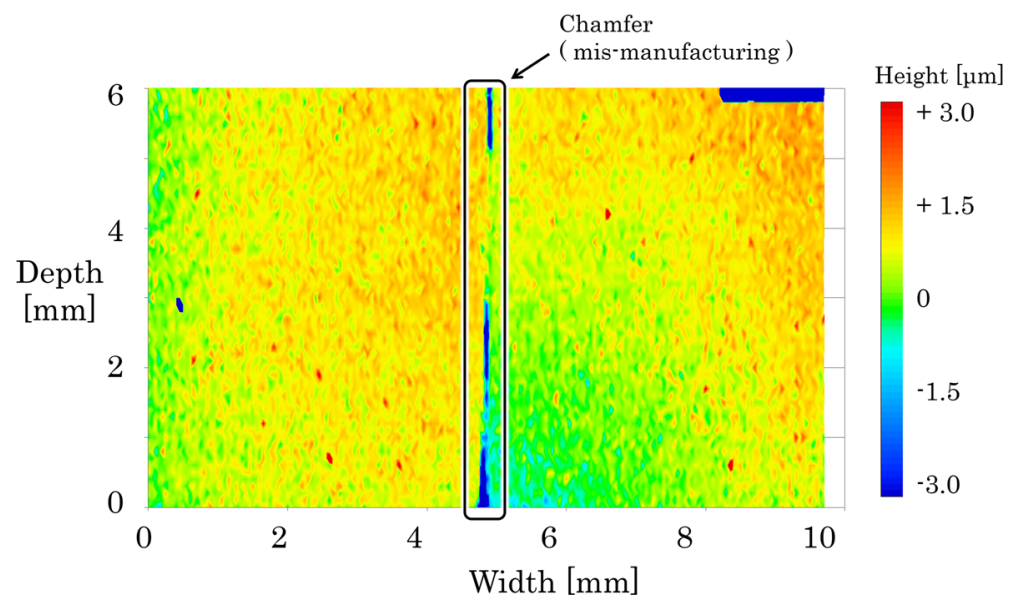

Fig. 14 Measured surface flatness of the waveguide absorber with an HAS flange 


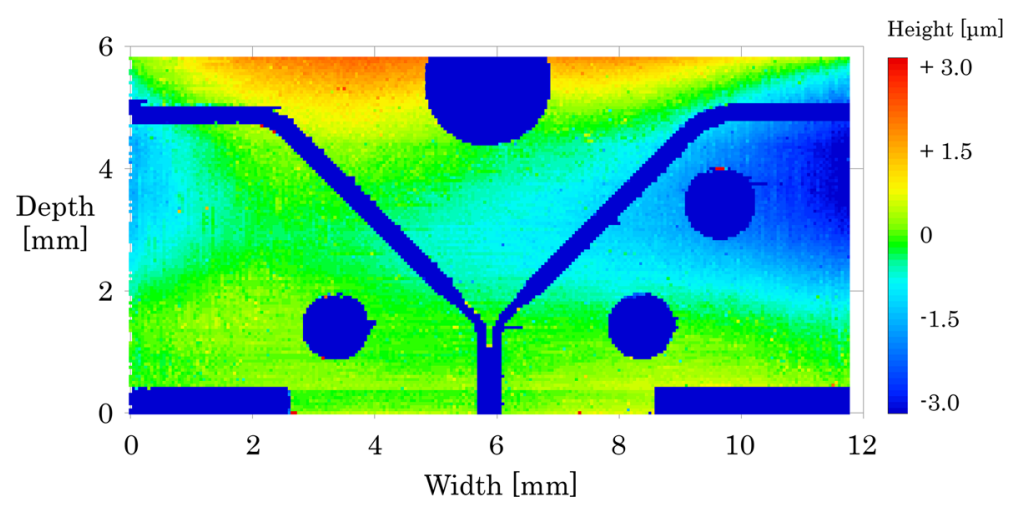

Fig. 15 Measured surface flatness of the 500-GHz band SST-CP two-divided surface

and planetary atmosphere observations. As shown in Table 1, the proposed SST-CP is considerably easier to handle and has higher performances than conventional circularly polarized wave separators including quasi-optical methods. For the SST-CP design, we applied the findings of the 230-GHz SST-CP device development, and developed a new manufacturing method to suppress the manufacturing errors, which would otherwise be too great. In addition, we developed a new original high-accuracy and very small (HAS) waveguide flange, which has a very small size of $10 \times 10 \mathrm{~mm}^{2}$ and a low horizontal positioning error of approximately $3 \mu \mathrm{m}$. Furthermore, to obtain very flat surfaces on blank materials, we developed a new high-accuracy mechanical polishing technique with a resin fixture. The 500GHz SST-CP manufactured by these new technologies realized an X-pol talk level of $-30 \mathrm{~dB}$ at $470-505 \mathrm{GHz}$ and $-20 \mathrm{~dB}$ at $430-510 \mathrm{GHz}$ in practice. In addition, the new HAS flange allows operation with almost no characteristic deterioration due to connection position errors. The surface flatness obtained by polishing with the resin fixture is very uniform within $3 \mu \mathrm{m}$, which is considered to effectively suppress the characteristic deterioration of the SST-CP. These results indicate that the development of the 500-GHz band SST-CP in this paper was successful, and it is expected that the new manufacturing techniques developed in this work will enable stable manufacture of higher-frequency waveguide devices, such as for the $1-\mathrm{THz}$ band.

Acknowledgments The authors would like to thank Satoshi Ochiai and Issei Watanabe of the National Institute of Information and Communications Technology (NICT) for measuring the developed SST-CP with a VNA and extender module at NICT. We are also very grateful to Masanori Ishino of Kawashima Manufacturing Co., Ltd. (KMCO) for conducting the high-precision machining of the SST-CP and HAS flange.

Table 1 The comparison table of circular polarizers in $\mathrm{mm} / \mathrm{sub}-\mathrm{mm}$ band

\begin{tabular}{|c|c|c|c|c|c|c|c|c|c|}
\hline $\begin{array}{l}\text { Circular } \\
\text { Polarizer } \\
\text { Type }\end{array}$ & $\begin{array}{l}\text { Adequate } \\
\text { frequency } \\
\text { band }[\mathrm{GHz}]\end{array}$ & $\begin{array}{c}\text { Fraetional } \\
\text { Bandwidth } \\
{[\%]}\end{array}$ & $\begin{array}{c}\text { Insertion } \\
\text { Losses }\end{array}$ & $\begin{array}{l}\text { Return } \\
\text { Losses } \\
\text { [dB] }\end{array}$ & $\begin{array}{c}\text { X-Pol } \\
\text { Talk } \\
{[\mathrm{dB}]}\end{array}$ & $\begin{array}{l}\text { Simple } \\
\text { Scaling } \\
\text { in band }\end{array}$ & $\begin{array}{c}\text { Size for } \\
\text { wavelength }\end{array}$ & $\begin{array}{l}\text { Aging } \\
\text { vary }\end{array}$ & Usability \\
\hline $\begin{array}{c}\text { SST-CPs } \\
\text { with thin septum }\end{array}$ & $\begin{array}{c}-86 \\
\text { (expected) }\end{array}$ & $\sim 15$ & Low & -20 & -30 & $\Delta$ & Small & No. & Easy \\
\hline $\begin{array}{l}230 \mathrm{CHz} \\
\mathrm{SSST} \cdot \mathrm{CP}[14]\end{array}$ & $\begin{array}{c}\sim 345 \\
\text { (expected) }\end{array}$ & 16 & Middlo & -20 & .25 & $\circ$ & Middlo & No. & Easy \\
\hline 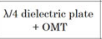 & -500 & 10 & High & -15 & $\cdot 15$ & $\Delta$ & Large & Yes. & Moderate \\
\hline $\begin{array}{c}\text { Wirggrid } \\
\text { interferometer }[15]\end{array}$ & 650 & $5 \sim 10$ & Low & $<-25$ & . & $x$ & Very Large & Yse. & Difficult \\
\hline $\begin{array}{l}\text { The proposod } \\
\text { now SST-CP }\end{array}$ & $\begin{array}{c}-1,000 \\
\text { (expected) }\end{array}$ & 16 & Low & -20 & 35 & 0 & Very Small & No. & Moderate \\
\hline
\end{tabular}


Funding This work was financially supported by JSPS KAKENHI Grant Number JP 17H07405.

Open Access This article is licensed under a Creative Commons Attribution 4.0 International License, which permits use, sharing, adaptation, distribution and reproduction in any medium or format, as long as you give appropriate credit to the original author(s) and the source, provide a link to the Creative Commons licence, and indicate if changes were made. The images or other third party material in this article are included in the article's Creative Commons licence, unless indicated otherwise in a credit line to the material. If material is not included in the article's Creative Commons licence and your intended use is not permitted by statutory regulation or exceeds the permitted use, you will need to obtain permission directly from the copyright holder. To view a copy of this licence, visit http://creativecommons.org/licenses/by/4.0/.

\section{References}

1. The Event Horizon Telescope Collaboration, "First M87 Event Horizon Telescope Results I: The Shadow of the Supermassive Black Hole", The Astrophysical Journal Letters, 875:L1 (17pp), 2019 April 10. https://doi.org/10.3847/2041-8213/ab0ec7

2. The Event Horizon Telescope Collaboration, "First M87 Event Horizon Telescope Results II: Array and Instrumentation”, The Astrophysical Journal Letters, 875:L2 (28pp), 2019 April 10. https://doi.org/10.3847 /2041-8213/ab0c96

3. Y. Yonekura, et al., "The Hitachi and Takahagi $32 \mathrm{~m}$ radio telescopes: Upgrade of the antennas from satellite communication to radio telescopes", Publ. Astron. Soc. Japan 68(5), 74(1-31), August 2013. https://doi.org/10.1093/pasj/psw045

4. Jonathan Weintroub, "A Submillimeter VLBI Array", Journal of Physics: Conference Series 131 (2008) 012047. https://doi.org/10.1088/1742-6596/131/1/01204

5. K. Harada, et al., "VLBI Direct Sampling Digital Frontend", IVS NICT Technology Development Center News, Proceedings of the 15th NICT TDC Symposium pp26-28, June 2016. http://www2.nict.go. jp/sts/stmg/ivstdc/news_36/tdc_news36.pdf

6. K. Furusawa, I. Morohashi, N. Sekine and I. Hosako, "A numerical study on a photonic assisted analog-todigital converter operating at $100 \mathrm{GSa} / \mathrm{s}$ ”, IVS NICT Technology Development Center News, Proceedings of the 16th NICT TDC Symposium pp1-4, 21 June 2017. http://www2.nict.go.jp/sts/stmg/ivstdc/news_37 /pctdc_news37.pdf

7. S. Asayama, et al. "Development of ALMA Band4 (125-163 GHz) receiver", Publ. Astron. Soc. Jpn (2014) 66 (3), 57(1-13). https://doi.org/10.1093/pasj/psu026

8. N. Satou, et al. "A Submillimeter Cartridge-Type Receiver ALMA Band 8 (385-500GHz) Qualification Model", Publications of the Astronomical Society of Japan, Volume 60, Issue 5, 25 October 2008, Pages 1199-1207, https://doi.org/10.1093/pasj/60.5.1199

9. Y. Fujii, et al., "The First Six ALMA Band 10 Receivers", IEEE Transactions on Terahertz Science and Technology, Vol. 3, Issue. 1, 39-49, Jan. 2013. https://doi.org/10.1109/TTHZ.2012.2236147

10. H. Ohyama, T. Nagahama, A. Mizuno, H. Nakane and H. Ogawa, "Observations of stratospheric and mesospheric $\mathrm{O}_{3}$ with a millimeter-wave radiometer at Rikubetsu, Japan”, Earth Planet Sp 68, 34 (2016). https://doi.org/10.1186/s40623-016-0406-4

11. J. Bornemann and V. A. Labay, "Ridge Waveguide Polarizer with Finite and Stepped-Thickness Septum", IEEE Trans. Microw. Theory Tech, vol.43 No.8, pp1782-1787, Aug. 1995. https://doi.org/10.1109 122.402260

12. Marc J. Franco, "A High-Performance Dual-Mode Feed Horn for Parabolic Reflectors with a SteppedSeptum Polarizer in a Circular Waveguide”, IEEE Antennas Propagation Magazine, Vol. 53, No.3, pp142-14, June 2011

13. C. A. Leal-Sevillano, K. B. Cooper, J. A. Ruiz-Cruz, J. R. Montejo-Garai and J. M. Rebollar, "A 225 GHz Circular Polarization Waveguide Duplexer Based on a Septum Orthomode Transducer Polarizer", IEEE Trans. THz Sci. Tech. vol.3, no.5, pp574-583, Sep. 2013. https://doi.org/10.1109/TTHZ.2013.2264317

14. Y. Hasegawa, , "A new approach to suppress the effect of machining error for waveguide septum circular polarizer at $230 \mathrm{GHz}$ band in radio astronomy", Journal of Infrared, Millimeter, and Terahertz Waves, 2017, 38, 638-652 (2017). https://doi.org/10.1007/s10762-017-0364-3

15. S. Ochiai et al., "Receiver Performance of the Superconducting Submillimeter-Wave Limb-Emission Sounder (SMILES) on the International Space Station," in IEEE Transactions on Geoscience and Remote Sensing, vol. 51, no. 7, pp. 3791-3802, July 2013, https://doi.org/10.1109/TGRS.2012.2227758 
16. T. Onishi et al.,"A 1.85-m mm-submm Telescope for Large-Scale Molecular Gas Surveys in 12CO, 13CO, and $\mathrm{C} 18 \mathrm{O}(\mathrm{J}=2-1)$ ", Publications of the Astronomical Society of Japan, Volume 65, Issue 4, 25 August 2013, 78. https://doi.org/10.1093/pasj/65.4.78

17. A. Nishimura, "Observational Study of the Physical Properties of Giant Molecular Clouds by the $1.85-\mathrm{m}$ Millimeter/Sub-millimeter Telescope", Ph. D. Thesis of Osaka Prefecture University, 14 Jan 2014. https://doi.org/10.24729/00000756

18. Y. Hasegawa, S. Asayama, R. Harada, K. Tokuda, K. Kimura, H. Ogawa, and T. Ohnishi, "Observational demonstration of a high image rejection SIS mixer receiver using a new waveguide filter at $230 \mathrm{GHz}$ ", Publications of the Astronomical Society of Japan, Volume 69, Issue 6, 1 December 2017, 91, https://doi. org/10.1093/pasj/psx098

19. T. Ohnishi, N. Mizuno, A. Mizuno, and Y. Fukui, "NANTEN2 Project: CO and CI Survey of the Local Group", Galaxies in the Local Volume. Astrophysics and Space Science Proceedings. Springer, Dordrecht, 30 April 2008. https://doi.org/10.1007/978-1-4020-6933-8 31

20. R. Enokiya, et al., "High-mass star formation in Orion B triggered by cloud-cloud collision: Merging molecular clouds in NGC 2024", Publications of the Astronomical Society of Japan, psaa049, 02 June 2020. https://doi.org/10.1093/pasj/psaa049

21. M. T. Chen, et al, "The Greenland telescope: Thule operations", Proceedings Volume 10700, Ground-based and Airborne Telescopes VII; 107000H (2018). https://doi.org/10.1117/12.2313378

22. C. C. Han, et al, "The first-light receivers for the Greenland Telescope", Proc. SPIE 10708, Millimeter, Submillimeter, and Far-Infrared Detectors and Instrumentation for Astronomy IX, 1070835 (9 July 2018). https://doi.org/10.1117/12.2313475

23. Y. Sekimoto, et al., "The Mt. Fuji submillimeter-wave telescope", Review of Scientific Instruments 71, 2895 (2000). https://doi.org/10.1063/1.1150709

24. Q. Jiao, et al., " Resolved neutral carbon emission in nearby galaxies: [C I] Lines as Total Molecular Gas Tracers", The Astrophysical Journal, Volume 880, Number 2, 2 August 2019. https://doi.org/10.3847/15384357/ab29ed

25. M. Hitschfeld, et al., "12 CO 4-3 and [CI] 1-0 in the centers of NGC 4945 and Circinus", Astronomy and Astrophysics, Vol. 479, No. 1, pp. 75-82, 18 December 2007 https://doi.org/10.1051/0004-6361:20078018

26. R. Larsson, et al., "Mars submillimeter sensor on microsatellite: sensor feasibility study", Geosci. Instrum. Method. Data Syst, vol. 7, pp331-341. https://doi.org/10.5194/gi-7-331-2018, 2018

27. J. Urban, et al., "Global observations of middle atmospheric water vapour by the Odin satellite: An overview”, Planetary and Space Science, Vol. 55, Issue 9, pp1093-1102, June 2007. https://doi. org/10.1016/j.pss.2006.11.021

28. A. R. Kerr, "Mismatch Caused by Waveguide Tolerances, Corner Radii, and Flange Misalignment", ELECTRONICS DIVISION TECHNICAL NOTE NO. 215,11 Jan 2010. http://citeseerx.ist.psu. edu/viewdoc/summary?doi=10.1.1.614.4764

Publisher's Note Springer Nature remains neutral with regard to jurisdictional claims in published maps and institutional affiliations. 